\title{
NON-LINEAR PROCESSES IN 1D QUANTUM SPIN ORDERED SYSTEM UNDER STRONG MAGNETIC FIELD PULSE
}

\author{
V.S. Borovikov, L.Yu. Gorelik, S.I. Kulinich and V.YU. PoPKov
}

B. Verkin Institute for Low Temperature Physics and Engineering Ukrainian Academy of Sciences, Lenin Av. 47, 310164 Kharkiv, Ukraine

(Received August 26, 1996; in final form November 26, 1996)

Investigation of an exactly integrable quantum model of $1 \mathrm{D}$ magnetic (which is equivalent to a massive fermion system) is presented. It has been shown that under certain conditions a spin system will not absorb power from the strong homogeneous magnetic field pulse.

PACS numbers: $75.30 . \mathrm{Mb}, 74.70 . \mathrm{Tx}$

Self-induced 1D magnetic transparency similar to the well-known optical self-induced transparency in two-level molecular gas has been predicted in our previous paper [1]. A resonant soliton caused by interaction between 1D quantum spin system and a pulse of a strong magnetic field was a result of exact solution of the problem. The effect was shown to take place in a system with a fixed configuration of parameters. In particular, we have found that self-induced transparency may take place in an anisotropic spin system with excitations of the Fermi type and with a linear dispersion law. This condition was found to be of great importance in Ref. [1].

In the present paper we analyze the role of dispersion law linearity for the above-mentioned effects. We investigate an exactly solvable 1D spin model, similar to the one referred in the paper [1], but with a gap in the dispersion law caused by the external constant magnetic field $h_{0}$. We have found a variable magnetic field time profile $h(t)$ for which the spin system has zero absorption energy. As it was shown in Refs. $[1,2]$, the existance of such a profile means that the proper resonant soliton exist. The relevant profile $h(t)$ has been constructed as a function of two free parameters.

The above-mentioned problem for the case of an anisotropic spin XY-chain under an external magnetic field $h(t)=h_{0}+h_{t}$ is briefly represented below. The model is described by the following Hamiltonian:

$$
H=H_{0}-\mu h(t) \sum_{n} S_{n}^{z}, \quad H_{0}=-\sum_{n}\left(J_{x} S_{n}^{x} S_{n+1}^{x}+J_{y} S_{n}^{y} S_{n+1}^{y}\right) .
$$


Here $S_{n}$ is a spin operator at the site $n$. We investigated the XY-model with a specific relation between the nearest neighbour exchange constants $\left(J_{x}=\right.$ $-J_{y}=J$ ). In the model under consideration, the dispersion law of excitations has a gap caused by a constant $h_{0}$ of the magnetic field $h(t)$. The pulse duration of the variable external field $h_{t}$ is assumed to be much smaller than the relaxation times in the system under consideration.

The Wigner transformation (see, e.g. Ref. [3]) enables us to describe this magnetic system in terms of the Fermi creation and annihilation operators $a_{n}^{+}$ and $a_{n}$. According to the paper [1] it is convenient to consider a two-sublattice system with the Fermi operators on even $\left(c(n) \equiv a_{2 n}\right)$ and odd $\left(d(n) \equiv a_{2 n+1}\right)$ sites of the chain. Using a transition from the discrete index $n$ to the continual variable value $x$ and from the Fermi operators $c(n)$ and $d(n)$ to the Fermi fields $c(x)$ and $d(x)$ one may rewrite the Hamiltonian (1) in continuous limit as follows:

$$
\begin{aligned}
& H=\int \mathrm{d} x\left(\Phi^{+}(x), \tilde{H} \Phi(x)\right), \quad \tilde{H}=V \sigma_{3} p+\mu \sigma_{2} h(t), \\
& \Phi(x) \equiv 2^{-1 / 2}\left(\begin{array}{c}
c(x)-\mathrm{i} d(x) \\
\mathrm{i} c(x)-d(x)
\end{array}\right), \quad V=2 J a, \quad p=\mathrm{i} \frac{\partial}{\partial x},
\end{aligned}
$$

where $\sigma_{i}$ is the Pauli matrix, $\Phi(x)$ - the two-component Fermi field operator, $\hbar=c=1$.

At $t \rightarrow-\infty$ and $h_{0}=0$ the density matrix $\rho(t)$ of the system is defined by the Liouville equation $i \frac{\partial}{\partial t} \rho(t)=[H, \rho]$ and has a form of an equilibri wum density matrix $\rho_{0}=\exp \left(-\beta H_{0}\right),(\beta=1 / T, T$ is temperature $)$. The expression

$$
\rho(t)=\sum_{\kappa} \omega_{\kappa}|\kappa t\rangle\langle\kappa t|
$$

satisfies the Liouville equation only if time dynamics of a ket-vector $|\kappa t\rangle$ is defined by the Schrödinger equation with the Hamiltonian $H$. Here $\omega_{\kappa}$ is the Boltzmann weight. We seek an expression for the ket-vector in the following form:

$$
|\kappa t\rangle=\int \mathrm{d} x \Psi_{\kappa}(x, t) a^{+}(x)|0\rangle, \quad a(x)|0\rangle=0 .
$$

In this case for the function $\Psi_{\kappa}(x, t)$ we have a non-stationary Schrödinger equation

$$
\mathrm{i} \frac{\partial}{\partial t} \Psi_{\kappa}(x, t)=\tilde{H} \Psi_{\kappa}(x, t)
$$

The operator $\widetilde{H}$ is determined by Eq. (2). The boundary condition are

$$
\begin{aligned}
& \Psi_{\kappa}(x, t \rightarrow+\infty)=\exp \left(\mathrm{i} \alpha_{\kappa}\right) \Psi_{\kappa}(x, t \rightarrow-\infty), \\
& \Psi_{\kappa}(x, t \rightarrow-\infty)=\exp \left(\mathrm{i} E_{\kappa} t\right) \Psi_{\kappa}(x) .
\end{aligned}
$$

As the external field is homogeneous, it is natural to represent $\Psi_{\kappa}(x, t)$ as

$$
\Psi_{\kappa}(x, t)=\exp (\mathrm{i} k x) \Psi_{\kappa}(t) .
$$

At this stage the problem can be formulated as a search for a reflectionless potential $h(t)$ analogous to the one in the spectral problem in the framework of the inverse scattering method

$$
\mathrm{i} \frac{\partial}{\partial t} \Psi_{\kappa}(t)=\left[k \sigma_{3}+h(t) \sigma_{2}\right] \Psi_{\kappa}(t)
$$


At $h_{0}=0$ and $h_{t}(|t| \rightarrow \infty)=0$ an elementary reflectionless one-parametrical potential takes the form

$$
h(t ; \gamma)=\operatorname{const} / \cosh (\gamma t),
$$

where $\gamma$ is a free parameter.

Formally, introduction of a constant magnetic field $h_{0}$ corresponds to the substitution $h(t) \rightarrow h_{0}+h_{t}$ only, and all the above-mentioned expressions are valid. However, in Eq. (4) we are interested in the only $h(t)$ that are equal not to zero at $|h| \rightarrow \infty$ but to a constant value $u_{0}$, defined by the value of an external magnetic field. The corresponding spectral problem has been considered for the non-linear Schrödinger equation with repulsion (see, for example, Ref. [4])

$$
\mathrm{i} \frac{\partial}{\partial t} u=-\frac{\partial^{2}}{\partial x^{2}} u-|u|^{2} u
$$

But there are two essential circumstances in Eq.(6) that are not satisfactory for our case: (i) the equation (6) is complex, so the quantity $u$ is of a complex value, (ii) the boundary conditions for the equation (6) have the following form:

$$
u= \begin{cases}u_{0}, & x \rightarrow-\infty, \\ u_{0} \exp i \eta, & x \rightarrow+\infty,\end{cases}
$$

where the quantity $\eta$ is defined by scattering data. For the problem under consideration the corresponding value $u$ should be real, and the condition $\eta=0$ is necessary to search the reflectionless potentials $u$. Under the above conditions we have found the exact solution of the equation (4) for the inverse scattering problem by the Mikhailov's method [5]. The required reflectionless potential is a two-parametrical one and can be written as

$$
\begin{aligned}
& h\left(t ; \gamma_{1}, \gamma_{2}\right)=h_{0}-\frac{\partial}{\partial t} \Omega\left(t ; \gamma_{1}, \gamma_{2}\right), \\
& \tan (\Omega / 2)=\frac{\delta \sin \gamma_{1} \sinh \theta-\epsilon \cosh \gamma_{2} \sin \nu}{\delta \cos \gamma_{1} \cosh \theta-\epsilon \sinh \gamma_{2} \cos \nu} .
\end{aligned}
$$

The free parameters $\gamma_{1}$ and $\gamma_{2}$ define the following values in the expression (7):

$$
\begin{aligned}
& \delta=h_{0} \frac{\sinh 2 \gamma_{2}}{\cosh 2 \gamma_{2}-\cos 2 \gamma_{1}}, \quad \theta=2 \epsilon t+\text { const }, \\
& \epsilon=h_{0} \frac{\sin 2 \gamma_{1}}{\cosh 2 \gamma_{2}-\cos 2 \gamma_{1}}, \quad \nu=2 \delta t+\text { const. }
\end{aligned}
$$

Thus, in the framework of the inverse scattering method, the variable magnetic field profile (potential) has been constructed as a function of two free parameters. They vary amplitude and duration of magnetic field pulse $h(t)=h_{0}+h_{t}$. The effect of self-induced transparency was shown to take place in Fermi systems with the non-linear dispersion law of excitations as well. An important remark should be made at this stage. At $h_{0} \rightarrow 0$, the transition from the two-parametrical reflectionless potential (at $\left.h_{0} \neq 0\right)$ to the one-parametrical potential (at $h_{0}=0$ ) is absent. It means, the obtained exact solutions relate to different classes of functions (see Eqs. (5), (7)), though both of the solutions have a common nature based 
on the nonlinear interaction between the external magnetic field pulse and the $1 \mathrm{D}$ quantum spin system.

This research was partly supported by the grant INTAS-93-633.

\section{References}

[1] A.E. Borovik, V.S. Borovikov, L.Yu. Gorelik, Acta Phys. Pol. A 85, 405 (1994).

[2] A.E. Borovik, V.S. Borovikov, A.M. Frishman, Phys. Lett. A 140, 436 (1989).

[3] S.A. Pikin, V.M. Tsukernik, Zh. Eksp. Teor. Fiz. 50, 1377 (1966).

[4] A.C. Newell, Solitons in Mathematics and Physics, SIAM, Philadelphia 1985.

[5] A.V. Mikhailov, Phys. Lett. A 92, 51 (1982). 